\title{
A proposito di ebbimo
}

\author{
Luca Serianni
}

PUBBLICATO: 28 MAGGIO 2019

\section{Quesito:}

Alcuni lettori chiedono il nostro parere sulla forma ebbimo per 'avemmo'.

\section{A proposito di ebbimo}

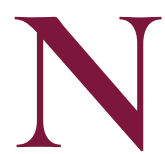

ei passati remoti dei verbi cosiddetti forti si ha un'alternanza tra le forme di prima, terza e sesta persona, accentate sulla radice, o rizotoniche (ébbi, ébbe, ébbero), e le forme di seconda, quarta e quinta, accentate sulla desinenza, o rizoatone (avésti, avémmo, avéste). Si tratta di un equilibrio instabile e come tale esposto a spinte livellatrici da parte della serie di impiego relativamente più frequente, quella rizotonica. Si tenga anche conto che l'uso del passato remoto è raro nell'italiano contemporaneo e, nella lingua parlata di vaste aree (tutto il Settentrione, ma anche parte dell'Italia centrale, a partire da Roma), è abitualmente sostituito dal passato prossimo: questo rende piu precaria la tenuta delle forme tradizionali.

Sono tipi ben noti sia ai dialetti (G. Rohlfs, nella sua Grammatica storica della lingua italiana e dei suoi dialetti, Torino, Einaudi, I967-I970, §584, cita un áppimu a Mascalucia, in Sicilia), sia alla tradizione letteraria. Come ha osservato Pier Vincenzo Mengaldo, L'epistolario di Nievo, Bologna, Il Mulino, I987, pp. 73-74, si tratta di forme "frequenti nella scripta ottocentesca non solo settentrionale".

Agli esempi di Nievo e del giovane Verga, citati o richiamati da Mengaldo, potremmo aggiungerne altri ricavati dagli archivi elettronici, relativi anche al Settecento e in un caso (Tesauro) al Seicento: Baretti, Bettinelli, Pietro Verri, Cuoco, D'Azeglio, Camillo Boito, D'Annunzio (Notturno), tutti per ebbimo; e ancora: Dossi e Svevo (seppimo), dissimo (Tommaseo prosatore), vidimo (Tesauro, Giannone, Tommaseo, Dossi). Del resto, anche nella letteratura del secondo Novecento il tipo ebbimo non è raro. Nel corpus di cento romanzi italiani allestito da Tullio De Mauro nel 2007 (Primo tesoro della lingua letteraria italiana del Novecento, Torino, UTET-Fondazione Bellonci) ricorrono 8 esempi, dal i947 al I976, in autori di varia provenienza regionale: Flaiano, Comisso, Chiara, Campanile, Cialente, contro 27 occorrenze complessive di avemmo, dal i95 al 200 I.

Non dobbiamo lasciarci ingannare dalle corrispondenti forme latine habuimus, diximus, vidìmus, che in realtà non hanno avuto continuazione diretta; sono ebbi, ebbe, ebbero, e cosi dissi, vidi ecc., che hanno modellato su di sé la quarta persona.

Ricostruire le vicende storiche di una forma non significa però legittimarne l'uso nella norma attuale. Dire o scrivere ebbimo qualificherebbe come poco istruito il parlante e lo scrivente di oggi. L'italiano contemporaneo, proprio per l'uso ormai condiviso delle sue strutture fondamentali, ha eliminato molte alternative che erano possibili fino all'altro ieri e sarebbe ingenuo richiamarsi a esempi di scrittori del passato più o meno recente (lo usavano Nievo, D'Annunzio e alcuni vincitori del premio Strega, e quindi sono autorizzato a fare lo stesso!). Nell'Ottocento anche persone colte potevano scrivere stó e nó: oggi li considereremmo badiali errori di ortografia; e come errore avvertiremmo anche un ebbimo in luogo dell'ormai affermato avemmo. 


\section{Cita come:}

Luca Serianni, A proposito di ebbimo , "Italiano digitale", 2019, IX, 2019/2 (aprile-giugno) DOI: $10.35948 / 2532-9006 / 2020.3119$

Copyright 2019 Accademia della Crusca

Pubblicato con licenza creative commons CC BY-NC-ND 